\title{
Predictive Value of CT Brain Perfusion Studies in Acute Ischemic Infarct Taking MRI Stroke Protocol As Gold Standard
}

\author{
Hafeez-ur-Rehman Junejo ${ }^{1}$, Shazia Yusuf ${ }^{2}$, Romasa Zeb ${ }^{3}$, Uswa Zeb ${ }^{4}$, Ahmed A. Zeb ${ }^{5}$, Aamena Ali ${ }^{2}$
}

1. Radiology, Bilawal Medical College, Jamshoro, PAK 2. Diagnostic Radiology, Capital Hospital, Islamabad, PAK 3. House Officer Medicine, Capital Hospital, Islamabad, PAK 4. Medicine, Capital Hospital, Islamabad, PAK 5. Medicine, Army Medical College, Rawalpindi, PAK

Corresponding author: Hafeez-ur-Rehman Junejo, dr.hafeez03@gmail.com

\section{Abstract}

\section{Background}

Acute ischemic stroke is the leading cause of serious chronic disability worldwide. Imaging plays a key role in early diagnosis and intervention, thus reducing mortality and morbidity related to ischemic stroke. Computed tomography (CT) perfusion study is a valuable imaging tool for the assessment of acute infarction. The objective of this study was to determine the predictive value of CT perfusion in diagnosing acute ischemic infarction taking Magnetic Resonance Imaging (MRI) stroke protocol (including Diffusion Weighted Imaging (DWI)) as a gold standard.

\section{Methods}

The cross-sectional validation study was conducted at a teaching hospital in Islamabad from June 2019 to December 2019. The study comprised a total of 125 patients of either gender with suspected acute ischemic stroke. The patients were scanned for CT perfusion and MRI stroke protocol on the same day. Scans were reported separately for the detection of acute ischemic infarction by the same consultant radiologist. The predictive value of CT perfusion was calculated accordingly.

\section{Results}

Of the 125 patients, $58 \%$ were male and $42 \%$ were female. The age of selected patients ranged between 38 to 70 years with a mean age of $56.12 \pm 9.69$ years. Acute ischemic infarction was detected in $86(69 \%)$ patients by CT perfusion study and in 120 (96\%) patients by MRI stroke protocol. The positive predicted value of CT perfusion for the detection of acute infarction was calculated as 98.83 and the negative predicted value was 10.25 .

\section{Conclusion}

Review began 06/11/2021 Review ended 07/08/2021 Published 07/20/2021

\section{(๑) Copyright 2021}

Junejo et al. This is an open access article distributed under the terms of the Creative Commons Attribution License CC-BY 4.0., which permits unrestricted use, distribution, and reproduction in any medium, provided the original author and source are credited.
CT perfusion study provides adequate sensitivity and specificity with good predictive value in the detection of acute ischemic infarct in stroke patients. This widely available and time-effective modality aids in the triage of patients for immediate endovascular intervention leading to maximal neurological benefit and improving outcomes.

Categories: Internal Medicine, Neurology, Radiology

Keywords: acute ischemic infarct, stroke, ct perfusion, mri, dwi

\section{Introduction}

Stroke is one of the common causes of morbidity and mortality throughout the world. It is the second major cause of death and the third major cause of disability worldwide [1]. The annual incidence of stroke in Pakistan is about 250/100,000 corresponding to 500,000 cases of stroke yearly [2,3]. An ischemic stroke is any thromboembolic or other event resulting in compromised blood flow to brain tissue [4]. The irreversibly damaged area of the cortex is the ischemic core and peripheral reversible zone of ischemia - "ischemic penumbra" [5]. The goal of the treatment is to restore flow to ischemic penumbra - the salvageable tissue around the infarct [6].

Tissue plasminogen activator (tPA) is a standard treatment in ischemic stroke patients [7]. Studies suggest the use of tPA along with timely endovascular reperfusion therapy can decrease disability to a greater extent and ensure normal or near-normal recovery [8]. The selection of candidates for endovascular reperfusion depends upon the determination of core to penumbra ratio [9]. The size of the penumbra is a predictor of neurologic outcome after reperfusion therapy. On the one hand, a large area of infarction with a small area of penumbra is considered a contraindication for endovascular reperfusion due to the risk of hemorrhage and low likelihood of a good outcome. On the other hand, the patients with larger penumbra can greatly 
benefit from the immediate therapy as it would lead to revascularization of the salvageable area and correspondingly decreased disability later in life $[10,11]$.

For the diagnosis of acute brain ischemic changes, MRI stroke protocol is a more sensitive and more accepted modality. MRI remains the gold standard for determining the infarct core [12]. However, MRI has a number of limitations and cannot be used in certain circumstances, i.e., irritable/restless patients and the patients having a metallic prosthesis. Another imaging modality with similar results in hyperacute stroke setting is CT perfusion. CT perfusion study predicts the infarct core rapidly and accurately with the ability to differentiate between irreversible infarcted core and reversible surrounding ischemic tissue - the penumbra [13].

Timely diagnosis is of primary importance in proceeding for intravenous thrombolysis and mechanical thrombectomy in hyperacute stroke patients [14]. CT perfusion studies are more suitable in the emergency setting to diagnose and select acute stroke patients that can benefit from endovascular reperfusion and thrombolytic therapy; due to its wide availability and considerably less time requirement $[15,16]$. This technique can be adapted in emergency facilities for acute stroke setting to facilitate diagnosis, aiding in establishing management plans of patients thus overall improving outcomes and optimizing expenses.

The purpose of this study was to determine the predictive value of CT perfusion in diagnosing acute ischemic infarct taking MRI stroke protocol (including Diffusion Weighted Imaging(DWI)) as a gold standard.

\section{Materials And Methods}

After approval from the institutional ethical review board, the present cross-sectional validation study was conducted at the Radiology Department of a teaching healthcare facility in Islamabad from June 2019 to December 2019. The sample size was calculated by using the WHO sample size calculator taking sensitivity of CT perfusion $69.9 \%$, specificity as $87.4 \%$, and prevalence of acute ischemic stroke $67 \%$, the estimated sample size was 125

The age of patients ranged between 38 - 70 years, male or female gender, suspected of acute ischemic stroke - those with symptoms of focal neurological deficit lasting for $\geqslant 24$ hours were included in the study. Those patients diagnosed with hemorrhagic or mixed (hemorrhagic + ischemic) stroke and those diagnosed with venous infarcts were excluded from the study. After informed consent, 125 patients with suspected acute ischemic stroke were included in the study using non-probability consecutive sampling. All the patients were entitled to medical treatment as the hospital belongs to a department of the federal government, and the employees and their families were entitled to free health services in the hospital. Hence the services of CT and MRI were free for these patients. The patients were scanned for CT perfusion and MRI stroke protocol on the same day.

A 64-slice spiral CT scanner machine (Toshiba, Minato City, Tokyo, Japan) was used to first perform noncontrast CT (NCCT) brain to exclude hemorrhage (hyperdense area) and large areas of clearly infarcted tissue (hypodense area); then one slice with suspected acute infarction was selected for CT perfusion study by the consultant radiologist with experience of $7+$ years. The scanning layer thickness was $8 \mathrm{~mm}$, matrix $512 \times 512$, tube voltage $120 \mathrm{kV}$, tube current $70 \mathrm{~mA}$, interval time: $1 \mathrm{sec}$; scanning time: $53 \mathrm{sec}$, a total of 80 layers, and scanning range was $80 \mathrm{~mm} .50 \mathrm{ml}$ of $370 \mathrm{mg} / \mathrm{ml}$ concentration of non-ionic iodine contrast agent (Inj. Lopromide) was injected through the right antecubital vein at a flow rate of 5.0 to $6.0 \mathrm{ml} / \mathrm{sec}$, followed by $50 \mathrm{ml}$ saline at 5.0 to $6.0 \mathrm{ml} / \mathrm{sec}$ and scanned at the same time (delay after injection was $7 \mathrm{sec}$ ). The scan was performed, and the CT image data was transmitted to the workstation, and Vitrea software (Toshiba, Minato City, Tokyo, Japan) was used for analysis. CT perfusion parameters, namely Cerebral Blood Flow (CBF), Cerebral Blood Volume (CBV), and Mean Transit Time (MTT), were measured. Acute infarction on perfusion study appeared as the area of the brain with prolonged relative MTT (> 145\%), markedly decreased CBF (<25 ml x $100 \mathrm{~g}-1$ x min-1), and markedly reduced CBV (<2 ml x $100 \mathrm{~g}-1)$ in comparison to the contralateral normal side.

MRI study was performed on a 1.5T MRI scanner (Canon Medical Systems, Otawara, Tochigi, Japan). The MRI stroke protocol employed included axial DWI, axial Apparent Diffusion Coefficient (ADC); axial, sagittal, coronal T2 weighted (T2W); axial, coronal Fluid Attenuated Inversion Recovery (FLAIR); and axial, sagittal, coronal T1 weighted (T1W) sequences. Acute ischemic infarction on MRI appeared as a hyperintense area on T2W/FLAIR sequences and had a characteristic appearance of the hyperintense area showing diffusion restriction (DWI) with the corresponding hypointensity on its ADC map. It remains hypointense on TIW images.

The same consultant radiologist reported both the CT perfusion and MRI scans separately. Findings of CT perfusion and MRI stroke protocol imaging were recorded for detection of acute ischemic infarct by both modalities. Data collected were entered and analyzed using SPSS version 22 (IBM Inc., Armonk, New York). Mean with standard deviation was calculated for quantitative variables like age. Frequency and percentage were calculated for gender. Sensitivity, specificity, positive predictive value, and negative predictive value 


\section{Cureus}

were calculated by a 2 × 2 table.

\section{Results}

Of the 125 patients, 73 (58\%) were male and 52 (42\%) were female. The minimum age was 38 years, and the maximum age was 70 years with a mean + standard deviation of $56.12 \pm 9.69$ years.

According to CT perfusion, acute ischemic infarction was detected in 86 (69\%) patients while the infarction was not detected in 39 (31\%) patients (Figure 1). According to MRI, acute ischemic infarction was detected in 120 (96\%) patients and the infarction was not detected in 5 (4\%) patients. The sensitivity of CT perfusion for detection of acute infarction was calculated as $70.83 \%$, the specificity was $80 \%$, the positive predicted value was 98.83 , the negative predicted value was 10.25 , and diagnostic accuracy was $71 \%$ (Table 1 ).

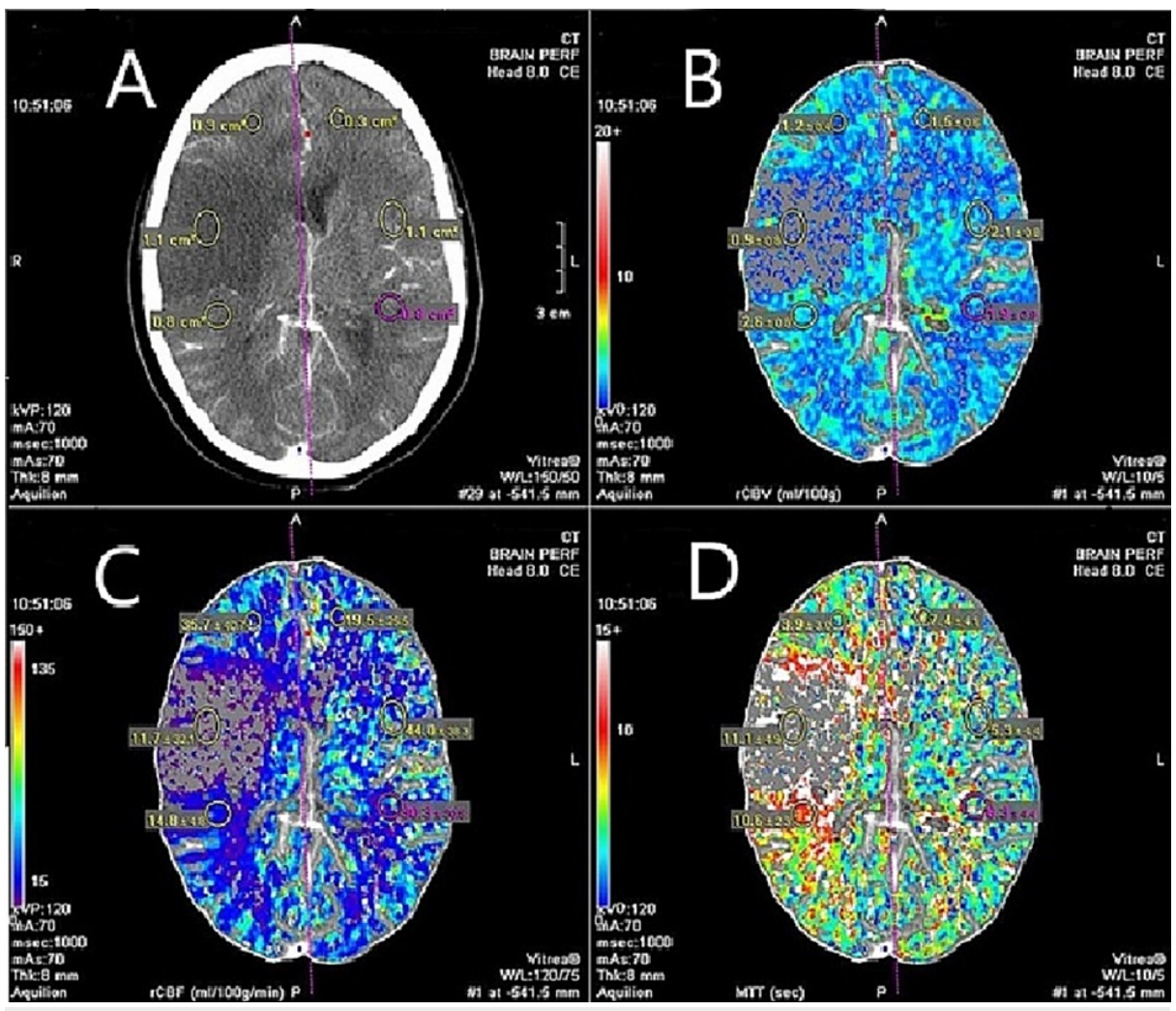

\section{FIGURE 1: CT Perfusion images}

A. Non-contrast axial CT brain image showing right-sided acute ischemic infarction with regions of interest selected on the infarcted side and contralateral normal side. Assessment of perfusion parameters suggestive of acute ischemic infarction B. Decreased Cerebral Blood Volume. C. Decreased Cerebral Blood Flow. D. Increased Relative Mean Transit Time.

\begin{tabular}{|l|l|l|}
\hline CT Perfusion & MRI-DWI Findings & \\
\hline & Acute Ischemic Infarct Detected & Acute Ischemic Infarct Not Detected \\
\hline Acute Ischemic Infarct Detected & $\mathrm{TP}=85$ & $\mathrm{FP}=1$ \\
\hline Acute Ischemic Infarct Not Detected & $\mathrm{FN}=35$ & $\mathrm{TN}=4$ \\
\hline Total & 120 & 5 \\
\hline
\end{tabular}

TABLE 1: 2×2 Table of CT Perfusion for detection of Acute Ischemic infarction

[TP=True Positive, TN=True Negative, FP=False Positive, FN=False Negative]

\section{Discussion}


According to our study, CT perfusion provides a significantly accurate assessment of ischemic brain tissue, including both core and penumbra, with high predictive value.

CT perfusion is a strong diagnostic modality for accurate assessment of volumetric core/penumbra mismatch ratios, thus predicting the volume of potentially rescuable tissue at risk that can be saved from recanalization therapy [17,18]. It not only aids in the selection of stroke patients for reperfusion treatment but also has good prognostic value [19]. CT may not only help in the selection of candidates for endovascular intervention in conjunction with tPA therapy, but it can also help in those patients in whom the use of tPA is contraindicated $[20,21]$.

CT perfusion is an attractive option to be adopted in health care setups, as it is a widely available, costeffective, and time-saving technique; for urgent diagnosis and early intervention of in-need hyperacute stroke patients. The early reperfusion of blocked arteries significantly improves the life quality of patients [22]. Thus, CT perfusion not only decreases overall mortality and morbidity but also significantly reduces the economic and social burden of a country [23].

According to a meta-analysis conducted in 2015, pooled overall sensitivity for CT perfusion technique was $55.7 \%$, whereas specificity was $92 \%$ [24]. Another study of 2017 concluded that sensitivity and specificity of CT perfusion scanning were $82 \%$ and $96 \%$, respectively. This study highlighted the superiority of CT perfusion study over non-contrast CT (NCCT) brain explaining that although NCCT brain can detect ischemic infarct in most cases, it is of limited value in detecting the early acute ischemic infarction (within 24 hours of the onset of symptoms) and it is influenced by severity and size of the infarct. CT perfusion is superior as it can detect early signs of ischemic stroke not visible on non-contrast CT scan [25].

CT perfusion study can be used to detect the acute ischemic infarction in health care setups where MRI facility is not available, or MRI is out of order due to some reason. The use of CT perfusion study can be of prime importance in those circumstances where MRI is contraindicated, like in patients with pacemakers, cochlear implants, or any other metallic prosthesis and for patients with claustrophobia [26]. Another advantage of CT perfusion study is the reduced time requirement as compared to MRI scanning; hence it can be employed as an emergency procedure in critically ill patients needing prompt intervention [27].

The disadvantages of CT perfusion studies include exposure to ionizing radiation, the use of iodinated contrast, and the inability of scanners to image the entire brain for perfusion imaging in many healthcare facilities, including our setup.

\section{Conclusions}

CT perfusion imaging provides high predictive value in the assessment of acute ischemic infarct in stroke patients. This widely available and time-effective modality aids in the triage of patients for immediate endovascular intervention leading to maximal neurological benefit and improving outcomes. This technique can be used as an alternative in cases of non-availability or contraindication of MRI.

\section{Additional Information}

\section{Disclosures}

Human subjects: Consent was obtained or waived by all participants in this study. Hospital ethics Committee, Capital Hospital Islamabad issued approval 1648. The committee hereby gives you unconditional permission to proceed with this project. Animal subjects: All authors have confirmed that this study did not involve animal subjects or tissue. Conflicts of interest: In compliance with the ICMJE uniform disclosure form, all authors declare the following: Payment/services info: All authors have declared that no financial support was received from any organization for the submitted work. Financial relationships: All authors have declared that they have no financial relationships at present or within the previous three years with any organizations that might have an interest in the submitted work. Other relationships: All authors have declared that there are no other relationships or activities that could appear to have influenced the submitted work.

\section{References}

1. Feigin VL, Norrving B, Mensah GA: Global burden of stroke. Circ Res. 2017, 120:439-48. 10.1161/CIRCRESAHA.116.308413

2. Khan M, Khan J, Ahmed S, Haq U: The epidemiology of stroke in a developing country (Pakistan) . J Neurol Stroke. 2018, 8:00275. 10.15406/jnsk.2018.08.00275

3. Khan MI, Khan JI, Ahmed SI, Ali S: The epidemiology of stroke in a developing country (Pakistan) . Pakistan Journal of Neurological Sciences. 2018, 13:30-44.

4. Huang Y, McNamara JO: Ischemic stroke: "acidotoxicity" is a perpetrator . Cell. 2004, 118:665-6. 10.1016/j.cell.2004.09.004

5. Lui YW, Tang ER, Allmendinger AM, Spektor V: Evaluation of CT perfusion in the setting of cerebral ischemia: patterns and pitfalls. Am I Neuroradiol. 2010, 31:1552-63. 10.3174/ainr.A2026

6. Srinivasan A, Goyal M, Al Azri F, Lum C: State-of-the-art imaging of acute stroke . Radiographics. 2006, 26 
Suppl 1:S75-95. 10.1148/rg.26si065501

7. Deb P, Sharma S, Hassan KM: Pathophysiologic mechanisms of acute ischemic stroke: An overview with emphasis on therapeutic significance beyond thrombolysis. Pathophysiology. 2010, 17:197-218. 10.1016/i.pathophys.2009.12.001

8. Jenson M, Libby J, Soule E, Sandhu SJ, Fiester PJ, Rao D: CT perfusion protocol for acute stroke expedites mechanical thrombectomy. Cureus. 2019, 11:e4546. 10.7759/cureus.4546

9. Sillanpaa N, Saarinen JT, Rusanen H, et al.: CT perfusion ASPECTS in the evaluation of acute ischemic stroke: thrombolytic therapy perspective. Cerebrovasc Dis Extra. 2011, 1:6-16. 10.1159/000324324

10. Hoeffner EG, Case I, Jain R, et al.: Cerebral perfusion CT: technique and clinical applications . Radiology. 2004, 231:632-44. 10.1148/radiol.2313021488

11. Ho CY, Hussain S, Alam T, Ahmad I, Wu IC, O'Neill DP: Accuracy of CT cerebral perfusion in predicting infarct in the emergency department: lesion characterization on CT perfusion based on commercially available software. Emerg Radiol. 2013, 20:203-12. 10.1007/s10140-012-1102-8

12. Copen WA, Yoo AJ, Rost NS, Morais LT, Schaefer PW, González RG, Wu O: In patients with suspected acute stroke, CT perfusion-based cerebral blood flow maps cannot substitute for DWI in measuring the ischemic core. PLoS One. 2017, 12:e0188891. 10.1371/journal.pone.0188891

13. Chiu AH, Phillips TJ, Phatouros CC, Singh TP, Hankey GJ, Blacker DJ, McAuliffe W: CT perfusion in acute stroke calls: a pictorial review and differential diagnoses. J Med Imaging Radiat Oncol. 2016, 60:165-71. $10.1111 / 1754-9485.12422$

14. de Lucas EM, Sánchez E, Gutiérrez A, et al.: CT protocol for acute stroke: tips and tricks for general radiologists. Radiographics. 2008, 28:1673-87. 10.1148/rg.286085502

15. Allmendinger AM, Tang ER, Lui YW, Spektor V: Imaging of stroke: Part 1, Perfusion CT--overview of imaging technique, interpretation pearls, and common pitfalls. Am J Roentgenol. 2012, 198:52-62. 10.2214/AJR.10.7255

16. Konstas AA, Goldmakher GV, Lee TY, Lev MH: Theoretic basis and technical implementations of CT perfusion in acute ischemic stroke, part 2: technical implementations. Am J Neuroradiol. 2009, 30:885-92. 10.3174/ajnr.A1492

17. Flottmann F, Broocks G, Faizy TD, et al.: CT-perfusion stroke imaging: a threshold free probabilistic approach to predict infarct volume compared to traditional ischemic thresholds. Sci Rep. 2017, 7:6679. 10.1038/s41598-017-06882-w

18. Schramm P, Schellinger PD, Klotz E, et al.: Comparison of perfusion computed tomography and computed tomography angiography source images with perfusion-weighted imaging and diffusion-weighted imaging in patients with acute stroke of less than 6 hours' duration. Stroke. 2004, 35:1652-8. 10.1161/01.STR.0000131271.54098.22

19. Xu S, Wang L, Zhao L: Clinical application value of brain CT perfusion imaging in the treatment of acute ischemic stroke thrombolytic therapy. Exp Ther Med. 2019, 17:3971-6. 10.3892/etm.2019.7431

20. Munich SA, Shakir HJ, Snyder KV: Role of CT perfusion in acute stroke management . Cor et Vasa. 2016, 58:215-24. 10.1016/j.crvasa.2016.01.008

21. Hana T, Iwama J, Yokosako S, et al.: Sensitivity of CT perfusion for the diagnosis of cerebral infarction . J Med Invest. 2014, 61:41-5. 10.2152/jmi.61.41

22. Biesbroek JM, Niesten JM, Dankbaar JW, Biessels GJ, Velthuis BK, Reitsma JB, van der Schaaf IC: Diagnostic accuracy of CT perfusion imaging for detecting acute ischemic stroke: a systematic review and metaanalysis. Cerebrovasc Dis. 2013, 35:493-501. 10.1159/000350200

23. Menon BK, Campbell BC, Levi C, Goyal M: Role of imaging in current acute ischemic stroke workflow for endovascular therapy. Stroke. 2015, 46:1453-61. 10.1161/STROKEAHA.115.009160

24. Xin Y, Han FG: Diagnostic accuracy of computed tomography perfusion in patients with acute stroke: a meta-analysis. J Neurol Sci. 2016, 360:125-30. 10.1016/j.jns.2015.11.046

25. Shen J, Li X, Li Y, Wu B: Comparative accuracy of CT perfusion in diagnosing acute ischemic stroke: A systematic review of 27 trials. PLoS One. 2017, 12:e0176622. 10.1371/journal.pone.0176622

26. Campbell BC, Christensen S, Levi CR, Desmond PM, Donnan GA, Davis SM, Parsons MW: Comparison of computed tomography perfusion and magnetic resonance imaging perfusion-diffusion mismatch in ischemic stroke. Stroke. 2012, 43:2648-53. 10.1161/STROKEAHA.112.660548

27. Bisdas S, Donnerstag F, Ahl B, Bohrer I, Weissenborn K, Becker H: Comparison of perfusion computed tomography with diffusion-weighted magnetic resonance imaging in hyperacute ischemic stroke. J Comput Assist Tomogr. 2004, 28:747-55. 10.1097/00004728-200411000-00004 